\title{
Activation of Liver X Receptor Attenuates Oleic Acid-Induced Acute Respiratory Distress Syndrome
}

\author{
Zanmei Zhao, ${ }^{\star \dagger}$ Dan $\mathrm{Xu}_{,}{ }^{\star \ddagger}$ Shuqiang $\mathrm{Li}^{\dagger}{ }^{\dagger}$ Bei He, ${ }^{\S}$ Yixian Huang, ${ }^{*}$ Meishu $\mathrm{Xu},{ }^{*}$ Songrong Ren, ${ }^{*}$ Song Li, ${ }^{*}$ Hui Wang, ${ }^{\ddagger}$ and \\ Wen $\mathrm{Xie}^{* \pi}$
}

From the Center for Pharmacogenetics and Department of Pharmaceutical Sciences* and the Department of Pharmacology and Chemical Biology, University of Pittsburgh, Pittsburgh, Pennsylvania; the Departments of Occupational Disease ${ }^{\dagger}$ and Respiratory Medicine, ${ }^{\S}$ Peking University Third Hospital, Beijing, China; and the Department of Pharmacology, ${ }^{\ddagger}$ School of Basic Medical Sciences, Wuhan University, Wuhan, China

\author{
Accepted for publication \\ June 23, 2016. \\ Address correspondence to \\ Wen Xie, M.D., Ph.D., Center \\ for Pharmacogenetics, Univer- \\ sity of Pittsburgh, 306 Salk \\ Pavilion, Pittsburgh, PA \\ 15261. E-mail: wex6@pitt.edu.
}

\begin{abstract}
Liver $X$ receptors (LXRs) were identified as receptors that sense oxidized cholesterol derivatives. LXRs are best known for their hepatic functions in regulating cholesterol metabolism and triglyceride synthesis, but whether and how LXRs play a role in the lung diseases is less understood. To study the function of LXRs in acute respiratory distress syndrome (ARDS), we applied the oleic acid (OA) model of ARDS to mice whose LXR was genetically or pharmacologically activated. The VP-LXR $\alpha$ knock-in (LXR-KI) mice, in which a constitutively activated $L X R \alpha(V P-L X R \alpha)$ was inserted into the mouse $L X R \alpha$ locus, were used as the genetic gain-of-function model. We showed that the $0 \mathrm{~A}$-induced lung damages, including the cytokine levels and total cell numbers and neutrophil numbers in the bronchoalveolar lavage fluid, the wet/dry weight ratio, and morphological abnormalities were reduced in the LXR-KI mice and wild-type mice treated with the LXR agonist GW3965. The pulmonoprotective effect of GW3965 was abolished in the LXRnull mice. Consistent with the pulmonoprotective effect of LXR and the induction of antioxidant enzymes by LXR, the OA-induced suppression of superoxide dismutase and catalase was attenuated in LXR-KI mice and GW3965-treated wild-type mice. Taken together, our results demonstrate that activation of LXRs can alleviate $\mathrm{OA}$-induced ARDS by attenuating the inflammatory response and enhancing antioxidant capacity. (Am J Pathol 2016, 186: 2614-2622; http://dx.doi.org/10.1016/j.ajpath.2016.06.018)
\end{abstract}

Acute lung injury (ALI), which manifests as bilateral pulmonary infiltrates, noncardiogenic pulmonary edema, respiratory distress, and hypoxemia, is a diffuse inflammatory process in the lung in response to a variety of disease conditions. ${ }^{1,2}$ Acute respiratory distress syndrome (ARDS) is a life-threatening condition of ALI. ALI and ARDS were first described by Ashbaugh et $\mathrm{al}^{3}$ in 1967. The prevalence of ARDS is a public health concern. ${ }^{4}$ The clinical management of ARDS is complex and challenging because the fundamental mechanisms that initiate and propagate the lung injury have not been fully understood.

The defining histopathological feature of ARDS is diffuse alveolar damage. This is characterized by severe alveolar epithelial and microvascular endothelial destruction and general polymorphonuclear leukocyte infiltration, along with intra-alveolar edema, hemorrhage, and fibrin deposition.
Hyaline membranes, which are depositions of cell debris and plasma proteins lining the alveolar wall, can be detected in persistent ARDS. ${ }^{6,7}$ Cytokines and proinflammatory mediators in the bronchoalveolar lavage (BAL) fluid or serum are reliable indicators of local or systemic inflammation and activation of polymorphonuclear leukocytes, monocytes, and macrophages.

Animal models are important in understanding the mechanisms of diseases, and these models are most useful if their characteristics are relevant to humans. The oleic acid

\footnotetext{
Supported in part by NIH grants ES023438 and DK083952 (W.X.), the National Natural Science Foundation of China Youth Science Fund Project 81402641 (Z.Z.), and the China Scholarship Council Visiting Scholarship 201506275099 (D.X.)

Disclosures: None disclosed.
} 
(cis-9-octadecenoic acid; OA)-induced lung injury represents a reliable model of ARDS. OA is the most common species of free fatty acid in mammals, representing $60 \%$ of the free fatty acid pool. When administered i.v., OA causes direct toxicity to the endothelial cells within 1 minute. Within 30 minutes, OA becomes detectable in the air spaces and induces endothelial necrosis and epithelial injury. It is believed that the OA-induced ARDS exhibits histopathological and physiological features similar to its human counterpart during both the acute and repair phases. ${ }^{11-13}$

Liver X receptors (LXRs) $\alpha(\mathrm{NR} 1 \mathrm{H} 3)$ and $\beta(\mathrm{NR} 1 \mathrm{H} 2)$ belong to the nuclear receptor family of transcription factors, which on ligand binding, stimulate the expression of their target genes. LXRs were identified as receptors for the oxidized cholesterol derivatives (oxysterols); therefore, they were also named oxysterol receptors. ${ }^{14}$ In addition, several synthetic LXR agonists, such as GW3965, have been developed. ${ }^{15}$ LXR $\alpha$ shows a high expression in the liver, lung, adipose, intestine, and kidney, whereas LXR $\beta$ is ubiquitously expressed. LXRs play an important role in cholesterol metabolism and triglyceride synthesis in various tissues. However, whether and how LXRs play a role in the pathophysiology of the lung is less understood. We and others reported that activation of LXR attenuated the lipopolysaccharide-induced acute lung injury. ${ }^{16,17}$

Herein, we used the OA-induced ARDS model to study the function of LXRs in lung inflammation and injury. We have uncovered a novel role for LXRs in alleviating the OAinduced ARDS. The pulmonary protective function of LXRs is likely because of the positive regulation of antiinflammatory and antioxidant genes.

\section{Materials and Methods}

Animals

Ten-week-old male and female wild-type (WT), LXR knock-in (LXR-KI), and LXR-DKO mice were used. The production of the LXR-KI mice by homologous recombination has previously been described. ${ }^{16}$ In brief, the LXRKI mice were generated by knocking-in the constitutively activated VP-LXR $\alpha$ into the mouse LXR $\alpha$ locus. VP-LXR $\alpha$ was generated by fusing the VP16 activation domain of the herpes simplex virus to the amino terminus of mouse $\operatorname{LXR} \alpha$ sequence. The homozygous LXR-KI mice express VP$\mathrm{LXR} \alpha$, whereas the expression of the endogenous LXR $\alpha$ is disrupted. The LXR-DKO mice, lacking both LXR $\alpha$ and LXR $\beta$, were a gift from Dr. David Mangelsdorf (University of Texas Southwestern Medical Center, Dallas, TX). All mice used are in the C56BL/6 background, except the LXRDKO mice, which are in the mixed background of C56BL/6 and SvJ129. The animal housing conditions and experimental procedures conformed to institutional regulations and were in accordance with the NIH guidelines on animal care. The institutional animal welfare committee approved all procedures.
Preparation of the $\mathrm{OA}$ Solutions

OA purchased from Sigma (St. Louis, MO) was used to prepare the $100 \mathrm{mmol} / \mathrm{L}$ Tris-oleate solution, as described. ${ }^{18}$ In brief, after weighting and water addition, the Tris-powder (Trizma base) was slowly added until the $\mathrm{pH}$ reached 10.0. This mixture was sonicated and after a complete oleate solubilization, the $\mathrm{pH}$ was carefully adjusted to 7.6 with diluted $\mathrm{HCl}$. The working solution of oleate was prepared by appropriate dilutions of the 100 $\mathrm{mmol} / \mathrm{L}$ Tris-oleate solution with sterile phosphate-buffered saline (pH 7.4).

\section{OA-Induced Lung Injury and LXR Ligand Treatment}

For the OA treatment, mice were subjected to tail vein injection of $0.15 \mathrm{~mL} / \mathrm{kg}$ OA or vehicle (Tris-HCl) in a total volume of $90 \mu \mathrm{L}$, and the mice were sacrificed 2 hours after. The 2-hour time point was chosen because previous studies showed that the concentrations of tumor necrosis factor (TNF)- $\alpha$ and IL- 6 in the BAL fluid peaked 1 to 3 hours after the OA application, ${ }^{19}$ and the tissue injury and an increase in the lung wet/dry weight ratio can also be assessed at this time point. ${ }^{20}$ When necessary, mice were subjected to a daily gavage of $20 \mathrm{mg} / \mathrm{kg}$ of the LXR agonist GW3965 in a total volume of $100 \mu \mathrm{L} 3$ days before the OA treatment.

\section{BAL Fluid Collection and Cell Counting}

These were performed essentially as described. ${ }^{21}$ In brief, 2 hours after the OA injection, mice were anesthetized by an i.p. injection of $150 \mathrm{mg} / \mathrm{kg}$ ketamine and $10 \mathrm{mg} / \mathrm{kg}$ xylazine. A small-caliber tube was inserted into the airway after the trachea was isolated by blunt dissection. The BAL fluids were collected by washing three times with $0.8 \mathrm{~mL}$ of sterile phosphate-buffered saline. The recovered BAL fluids were centrifuged at $1400 \times g$ for 10 minutes. The total BAL cells in the BAL fluid pellets were counted using a hemocytometer. The polymorphonuclear neutrophils were selectively identified and counted after staining with the May-Grunewald-Giemsa solution from Sigma. The cellfree supernatants of the BAL fluids were collected and subjected to the measurement of protein concentration using a BCA protein assay kit from Pierce/Thermo Fisher Scientific (Pittsburgh, PA).

\section{Measurement of the Lung W/D Ratio}

Mice were sacrificed and lungs were excised 2 hours after the OA treatment. Blood was removed by blotting the lungs with filter papers until dry, and the lungs were then immediately weighed. The lungs were subsequently placed in an oven at $65^{\circ} \mathrm{C}$ for 72 hours, and the dry weight was recorded. The lung wet/dry weight (W/D) ratio was calculated to assess tissue edema. 
Measurement of TNF- $\alpha$ and IL- 6 in the BAL Fluid and Serum

These were measured using enzyme-linked immunosorbent assay kits from Pierce/Thermo Fisher Scientific, according to the manufacturer's instructions.

\section{Measurement of SOD and CAT Activities}

To measure the superoxide dismutase (SOD) and catalase (CAT) activities, lungs were excised and washed thoroughly with phosphate-buffered saline to remove most of the blood contamination, then stored at $-80^{\circ} \mathrm{C}$ until used. Lungs were homogenized in $50 \mathrm{mmol} / \mathrm{L}$ potassium phosphate $(\mathrm{pH} 7.0)$ and $1 \mathrm{mmol} / \mathrm{L}$ ethylene diamine tetra-acetic acid. The homogenates were clarified by centrifugation at $10,000 \times g$ for 30 minutes at $4{ }^{\circ} \mathrm{C}$, and the supernatants were immediately used for the measurements of SOD and CAT activities using assay kits from Cayman Chemical (Ann Arbor, MI). The SOD activity was determined using a nitroblue tetrazolium reduction method for the detection of superoxide radicals generated by xanthine oxidase and hypoxanthine. The absorbance was monitored at 440 to $460 \mathrm{~nm}$. The CATC activity was measured by the rate of decrease in hydrogen peroxide absorbance at $540 \mathrm{~nm}$ and defined $1 \mathrm{U}$ as the amount of enzyme that will cause the formation of $1.0 \mathrm{nmol}$ of formaldehyde per minute at $25^{\circ} \mathrm{C}$. The CAT activity was expressed as $\mathrm{nmol} / \mathrm{minute} / \mathrm{mL}$.

\section{Histology and Immunohistochemistry}

The lungs were freshly harvested and fixed in $10 \%$ neutralbuffered formalin for 24 hours. The tissues were histologically processed, embedded in paraffin, divided into sections (4 $\mu \mathrm{m}$ thick), and stained with hematoxylin and eosin. For immunohistochemistry, the paraffin sections were deparaffinized and rehydrated. The endogenous peroxidase activity was blocked by incubating the sections in $3 \% \mathrm{H}_{2} \mathrm{O}_{2}$ solution in methanol. Citrate buffer incubation was used to unmask the antigens. The slides were incubated in blocking buffer in a humidified chamber for 1 hour before incubation with the primary anti-myeloperoxidase (MPO) antibody (catalog number AB9353, dilution at 1:25) from Abcam (Cambridge, MA), or the anti-8-hydroxyguanosine antibody (catalog number $\mathrm{AB} 10802$, dilution at 1:400) also from Abcam, for 1 hour. The slides were then washed and incubated in biotinylated secondary antibody and conjugates before the color reaction using $3,3^{\prime}$-diaminobenzidine tetrahydrochloride as the substrate. The slides were counterstained by hematoxylin.

\section{Statistical Analysis}

The results are expressed as the means \pm SD. One-way analysis of variance and Tukey's test were used for statistical analysis using GraphPad Prism software version 6.0
(GraphPad Software, Inc., La Jolla, CA). $P<0.05$ was considered statistically significant.

\section{Results}

Generation of LXR-KI Mice that Bear the Constitutive Activation of LXR $\alpha$ in the Lung

We have previously reported that both LXRs $\alpha$ and $\beta$ are abundantly expressed in the mouse lung. ${ }^{16}$ Immunohistochemical analysis showed that $\operatorname{LXR} \alpha$ is expressed in both the type I and type II lung epithelial cells. ${ }^{16}$ To study the in vivo functions of $\mathrm{LXR} \alpha$, we generated $\mathrm{LXR} \alpha$ knock-in mice that express a constitutively activated $\mathrm{LXR} \alpha$ (VP$\mathrm{LXR} \alpha$ ) in tissues that express the endogenous $\operatorname{LXR} \alpha$, including the lung. ${ }^{16}$ The VP-LXR $\alpha$ cDNA was generated by fusing the VP16 activation domain of the herpes simplex virus to the amino terminus of mouse $\operatorname{LXR} \alpha$ sequence (Figure 1A). To generate the targeting construct, VP-LXR $\alpha$ cDNA was placed in-frame and immediately after the endogenous ATG start codon of the mouse LXR $\alpha$ locus. After the homologous recombination, the sequence spanning part of exon 2, exons 3 to 7 , and the introns in between were replaced by VP-LXR $\alpha$. As such, VP-LXR $\alpha$ will be expressed under the control of the endogenous LXR $\alpha$ promoter, whereas the WT LXR $\alpha$ will be disrupted in the homozygous LXR-KI mice. The expression of VP-LXR $\alpha$ in the LXR-KI mice was confirmed by Northern blot analysis. The expression of VP-LXR $\alpha$ in the LXR-KI mice was detected in the lung, liver, and small intestine, a panel of tissues known to express LXR $\alpha$ (Figure 1B). In contrast, the endogenous $\mathrm{LXR} \alpha$ transcript in these three tissues was detected only in the WT but not in the LXR-KI mice. Little expression of VP-LXR $\alpha$ was detected in the brain of the LXR-KI mice, consistent with the known low expression of this LXR isoform in the brain of the WT mice (Figure 1B).

\section{Activation of LXR Attenuates the OA-Induced Increases in Pulmonary Permeability and Edema}

To determine the effect of LXR activation on the OAinduced ARDS, we first treated male WT and LXR-KI mice with OA by tail vein injections. Two hours later, mice were sacrificed and the lung W/D ratio, BAL fluid protein concentrations, and lung histology were evaluated. The lung W/D ratio is widely used to assess pulmonary vascular permeability, which is a marker of damage to the capillary membrane and one of the key features of experimental ARDS. $^{22}$ Treatment of WT mice with OA resulted in an expected increase in the lung W/D ratio. In a sharp contrast, the OA-induced lung W/D ratio increase in LXR-KI mice was reduced to approximately half of the OA-treated WT mice (Figure 2A). A significantly decreased lung W/D ratio was also observed in male WT mice pretreated with the synthetic LXR agonist GW3965 for 3 days (Figure 2A). However, the protective effect of GW3965 in OA-treated 
A

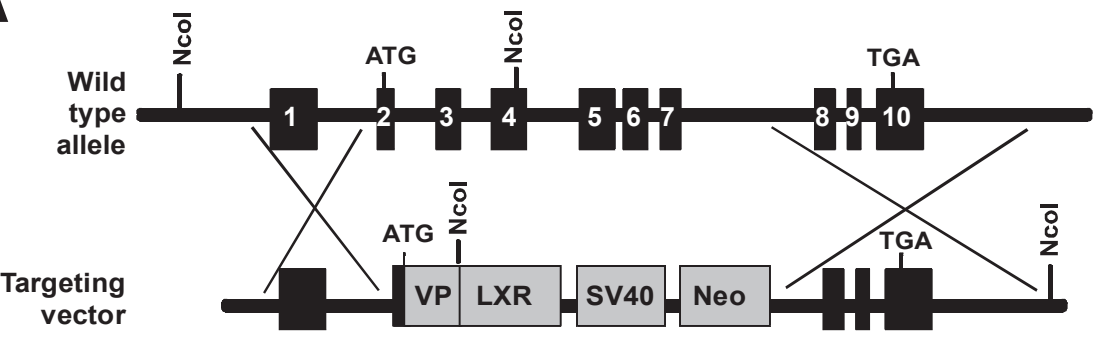

Figure 1 Generation of liver $X$ receptor (LXR) $\alpha$ knock-in (LXR-KI) mice that bear the constitutive activation of LXR $\alpha$ in the lung. A: Strategy to knock-in VP-LXR $\alpha$ into the mouse LXR $\alpha$ locus. The positions and numbers of the $L X R \alpha$ gene exons are labeled. On gene targeting, part of exon 2, exons 3 to 7 , and introns in between of the wild-type (WT) allele are replaced by the VP-LXR $\alpha$-SV40-Neo cassette. B: The expression of knock-in VP-LXR $\alpha$ and the endogenous $\operatorname{LXR} \alpha$ was determined by Northern blot analysis using a $\operatorname{LXR} \alpha$ cDNA probe. Ethidium bromide staining of the agarose gel is to show the sample loading.
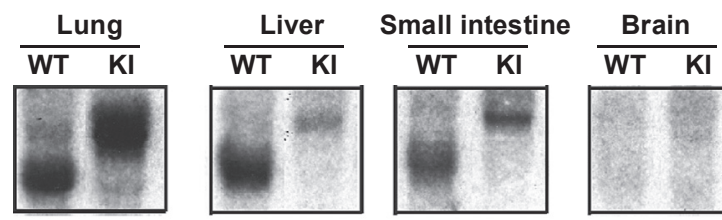

B
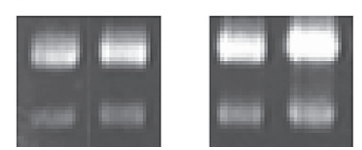

VP-LXR $\alpha$

LXRa
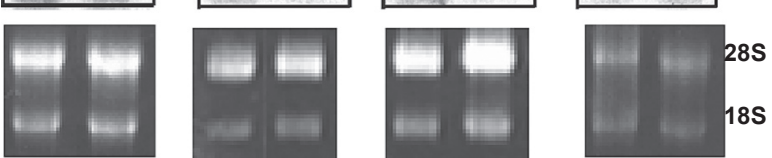

mice was abolished in mice deficient of both $\operatorname{LXR} \alpha$ and LXR $\beta$ (LXR-DKO) (Figure 2A). A similar pattern of the inhibitory effect of the LXR-KI allele and GW3965 treatment was observed when the protein concentrations were measured in the BAL fluid (Figure 2B). At the histological level, the OA-induced pulmonary interstitial edema and infiltration of cells into the interstitium and alveolar spaces observed in the WT mice was attenuated in the LXR-KI
A

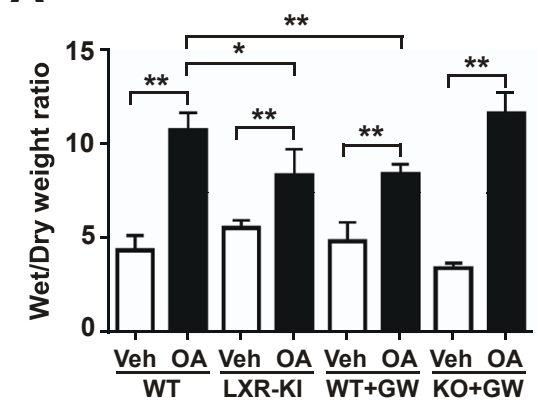

C
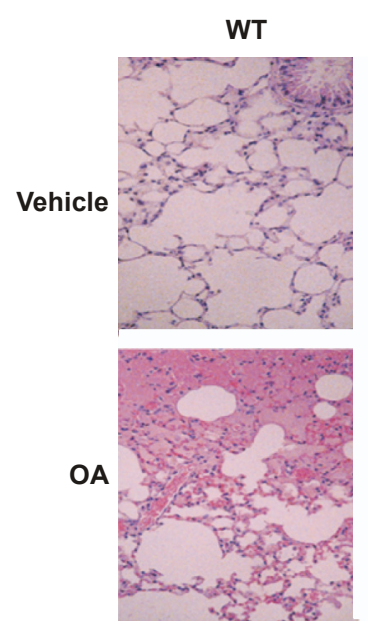

B

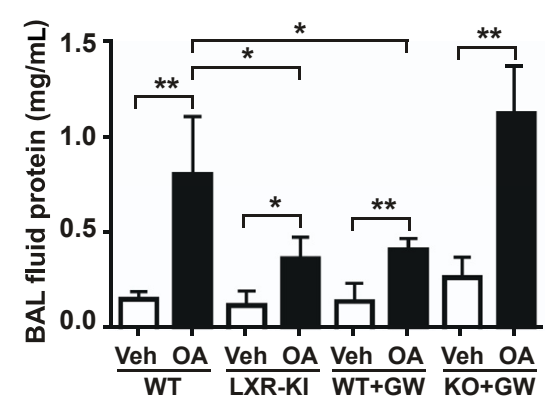

LXR-KI
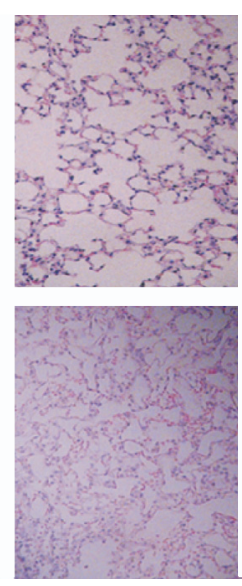
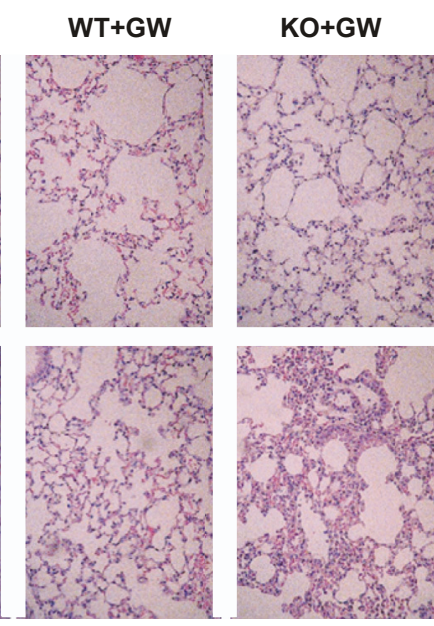

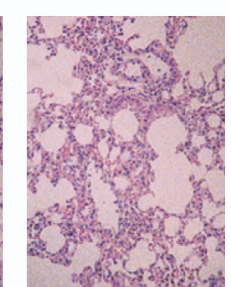

Figure 2 Activation of liver $X$ receptor (LXR) attenuates the oleic acid $(O A)$-induced increases in pulmonary permeability and edema. All mice are males. Wild-type (WT), LXR knock-in (LXR-KI), and LXR-DKO [knockout (KO)] mice received a single tail vein injection of vehicle (Veh) or OA. When necessary, WT and LXR-DKO mice were treated with GW3965 (GW; daily i.p. injections at $20 \mathrm{mg} / \mathrm{kg}$ ) for 7 days before being treated with Veh or OA. Mice were sacrificed 2 hours after the $O A$ treatment and analyzed. A: The wet/dry weight ratio of the lung tissues. B: The protein concentrations in the cellfree bronchoalveolar lavage (BAL) fluid supernatants. C: Hematoxylin and eosin staining of the lung sections. $n=8$ (Veh group, $\mathbf{A}$ and $\mathbf{B}$ ); $n=11$ (WT + OA group, $\mathbf{A}$ and $\mathbf{B}$ ) $; n=4$ (other groups, $\mathbf{A}$ and $\mathbf{B}) .{ }^{*} P<0.05,{ }^{*} P P<0.01$. Original magnification, $\times 200(C)$. 
mice and WT, but not LXR DKO, mice treated with GW3965 (Figure 2C). A similar pattern of attenuation of the OA-responsive increase in pulmonary permeability and edema was observed in the female LXR-KI mice (Supplemental Figure S1), suggesting the protective effect of LXR on OA-induced ARDS was not sex specific.

\section{Activation of LXR Attenuates the OA-Induced Infiltration of Neutrophils}

Another hallmark of ARDS, including those induced by $\mathrm{OA}$, is the infiltration of neutrophils. ${ }^{13}$ As expected, treatment of WT male mice with OA resulted in dramatic increases in the total cell numbers (Figure 3A) and neutrophil numbers (Figure $3 \mathrm{~B}$ ) in the BAL fluid. In contrast, the OAinduced total cell and neutrophil number increases in the BAL fluid of the LXR-KI mice were reduced to approximately $50 \%$ of the OA-treated WT mice (Figure 3, A and B). The decreased BAL fluid cell and neutrophil numbers were also observed in WT mice treated with GW3965, and the GW3965 effect was abolished in the LXR-DKO mice (Figure 3, A and B). The LXR-DKO mice, in the absence of GW3965 and compared to their WT counterparts, also showed heightened sensitivity to OA (Figure 3, A and B). To further confirm the neutrophil infiltration, we performed immunohistochemical staining of the MPO, a widely used index of polymorphonuclear neutrophil sequestration that reflects the infiltration of lung parenchymal phagocytes. ${ }^{23}$ Treatment with OA increased the immunostaining of MPO in the lung, and this effect was attenuated in LXR-KI mice and WT mice treated with GW3965 (Figure 3C). Again, the GW3965 effect in reducing the MPO immunostaining was abolished in LXR-DKO mice. The LXR-DKO mice, in the absence of GW3965, also showed increased immunostaining of MPO (Figure 3C). A similar pattern of attenuation of OA-responsive increase in neutrophil infiltration was observed in the female LXR-KI mice (Supplemental Figure S2).
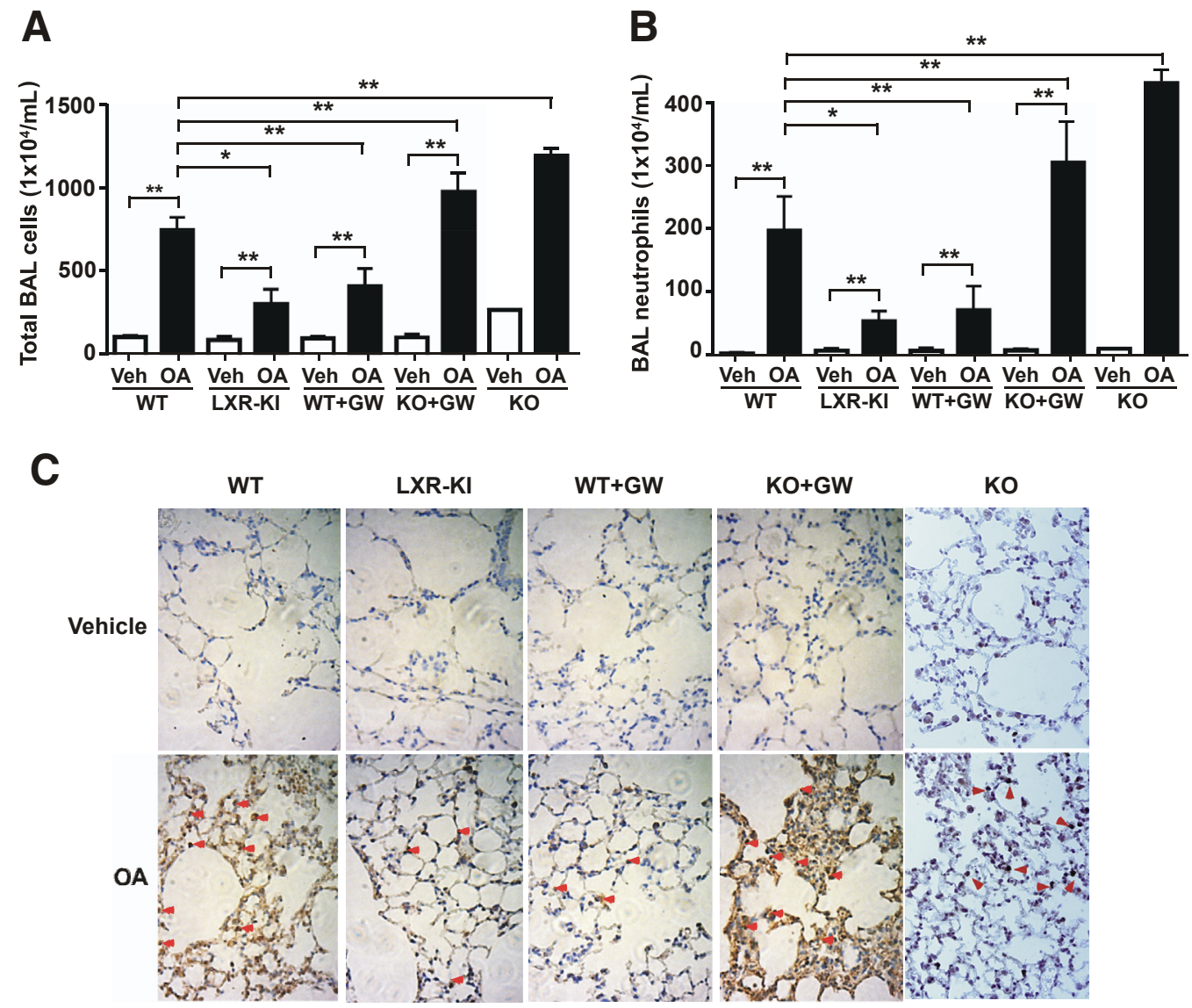

Figure 3 Activation of liver X receptor (LXR) attenuates the oleic acid (OA)-induced infiltration of neutrophils. Wild-type (WT), LXR knock-in (LXR-KI), and LXR-DKO [knockout (KO)] mice received a single tail vein injection of vehicle (Veh) or OA. When necessary, WT and LXR-DKO mice were treated with GW3965 (GW; daily i.p. injections at $20 \mathrm{mg} / \mathrm{kg}$ ) for 7 days before being treated with Veh or OA. Mice were sacrificed 2 hours after the $0 \mathrm{~A}$ treatment and analyzed. Last two groups of LXR DK0 mice were not treated with GW3965. A: The total bronchoalveolar lavage (BAL) cell numbers were counted using a hemocytometer. B: The polymorphonuclear neutrophils were stained with the May-Grunwald and Giemsa solution, and their cell numbers were counted. C: Immunohistochemical staining of the lung paraffin sections using the anti-myeloperoxidase (MPO) antibody. Arrowheads indicate the positive MP0 staining. $n=8$ (Veh group, A and $\mathbf{B}$ ) $; n=11$ (WT + OA group, A and B) $; n=4$ (other groups, $\mathbf{A}$ and $\mathbf{B}$ ). ${ }^{*} P<0.05,{ }^{*} P<0.01$. Original magnification, $\times 400$ (C). 


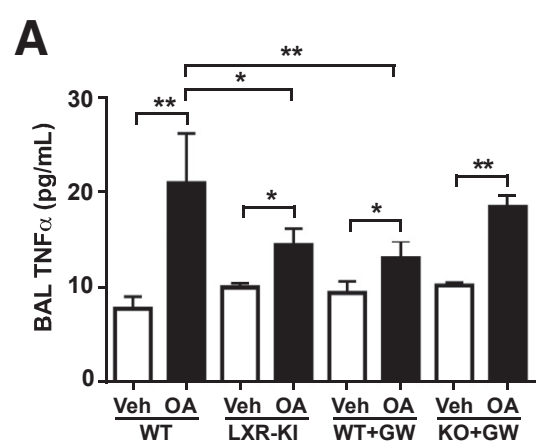

B

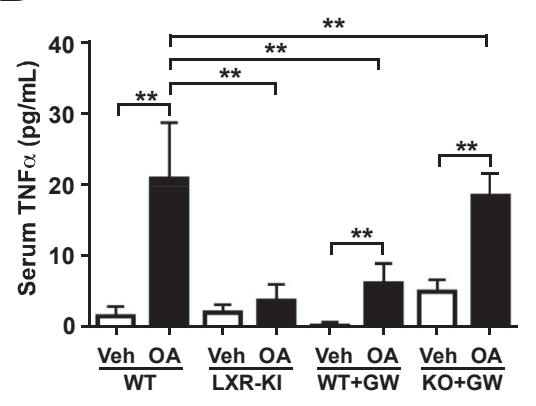

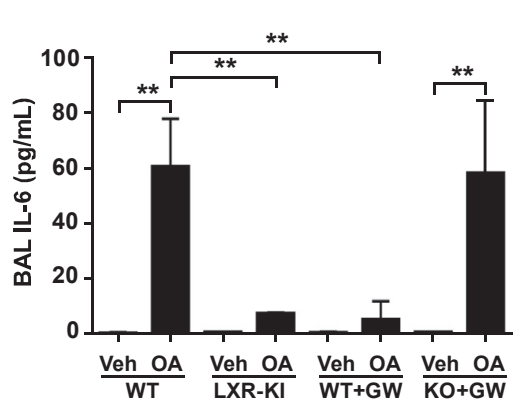

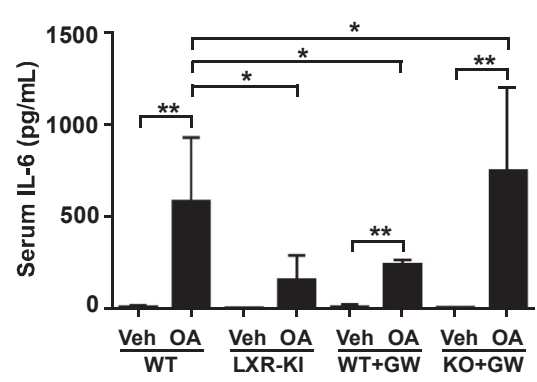

Figure 4 Activation of liver $X$ receptor (LXR) attenuates the oleic acid (OA)-induced local and systemic inflammation. Wild-type (WT), LXR knock-in (LXR-KI), and LXR-DKO [knockout (KO)] mice received a single tail vein injection of vehicle (Veh) or OA. When necessary, WT and LXR-DKO mice were treated with GW3965 (GW; daily i.p. injections at $20 \mathrm{mg} / \mathrm{kg}$ ) for 7 days before being treated with Veh or $0 \mathrm{~A}$. The mice were sacrificed before the bronchoalveolar lavage (BAL) fluid and the blood was collected. A: The concentrations of tumor necrosis factor (TNF) $-\alpha$ and IL- 6 in the cellfree BAL fluid supernatants. B: The concentrations of TNF- $\alpha$ and IL- 6 in the serum. $n=8$ (Veh group); $n=11$ (WT + OA group); $n=4$ (other groups). ${ }^{*} P<0.05,{ }^{*} P<0.01$.

\section{Activation of LXR Attenuates the OA-Induced Local and Systemic Inflammation}

A major pathological event associated with ARDS is local and systemic inflammation. The local and systemic inflammation was evaluated by measuring the levels of TNF- $\alpha$ and IL-6 in the BAL fluid and serum, respectively. Indeed, treatment of WT male mice with OA resulted in significantly increased levels of TNF- $\alpha$ and IL- 6 in the BAL fluid (Figure 4A) and in the serum (Figure 4B). The OA effect in increasing TNF- $\alpha$ and IL-6 levels in the BAL fluid (Figure 4A) and in the serum (Figure 4B) was substantially attenuated in the LXR-KI mice and the WT mice treated with GW3965. In contrast, the GW3965-treated LXR-DKO mice remained sensitive to OA in inducing the TNF- $\alpha$ and IL-6 levels (Figure 4, A and B). A similar pattern of attenuation of OA-responsive increase in local and systematic inflammation was observed in the female LXR-KI mice (Supplemental Figure S3).

\section{The Protective Effect of LXR Is Associated with Attenuation of OA-Induced Oxidative Stress and Induction of Antioxidant Genes}

Oxidative stress plays an important role in the development of OA-induced ARDS. ${ }^{24}$ Intracellular oxidative stress can lead to the generation of nucleic acid oxidation products, such as the 8-hydroxyguanosine, which has been widely used as a biomarker of oxidative stress. ${ }^{25}$ Indeed, we showed that treatment with $\mathrm{OA}$ increased the immunostaining of 8hydroxyguanosine in the lung, and this effect was attenuated in LXR-KI mice and WT mice treated with GW3965. The GW3965 effect in reducing the 8-hydroxyguanosine immunostaining was abolished in LXR-DKO mice (Figure 5A). In understanding the protective effect of LXR activation, we found the lung expression of several antioxidant genes, such as the glutathione $S$-transferase $\mathrm{m} 1$ (Gstml; Figure 5B) and Gstpl (Figure 5C), was increased in both the LXR-KI mice and the GW3865-treated WT mice in the absence of OA challenge, consistent with our previous report. ${ }^{16}$ Interestingly, the expression of both Gst isoforms was decreased when mice were treated with OA regardless of the LXR activation, which might be secondary to the tissue damage and responses to the tissue damage. We then measured the activities of SODs and CAT that indicate the overall oxidative stress. SODs and CAT are direct antioxidants. SODs catalyze the dismutation of the superoxide anion to molecular oxygen and hydrogen peroxide. ${ }^{26,27}$ CAT is a ubiquitous antioxidant enzyme that is involved in the detoxification of hydrogen peroxide, a reactive oxygen species that causes cellular damages. We found that treatment of male WT mice with OA resulted in significant suppressions of the activities of SODs (Figure 5D) and CAT (Figure 5E) in the lung, and these suppressions were attenuated in the male LXR-KI mice and WT mice treated with GW3965. The effect of GW3965 in attenuating the suppression of SOD and CAT was abolished in the LXR-DKO mice (Figure 5, D and E). A similar pattern of attenuation of suppression of SOD and CAT was observed in the female LXR-KI mice (Supplemental Figure S4).

\section{Discussion}

Herein, we demonstrated the preventive effect of LXR on ARDS. Genetic or pharmacological activation of LXR effectively attenuated the OA-induced pulmonary edema, 


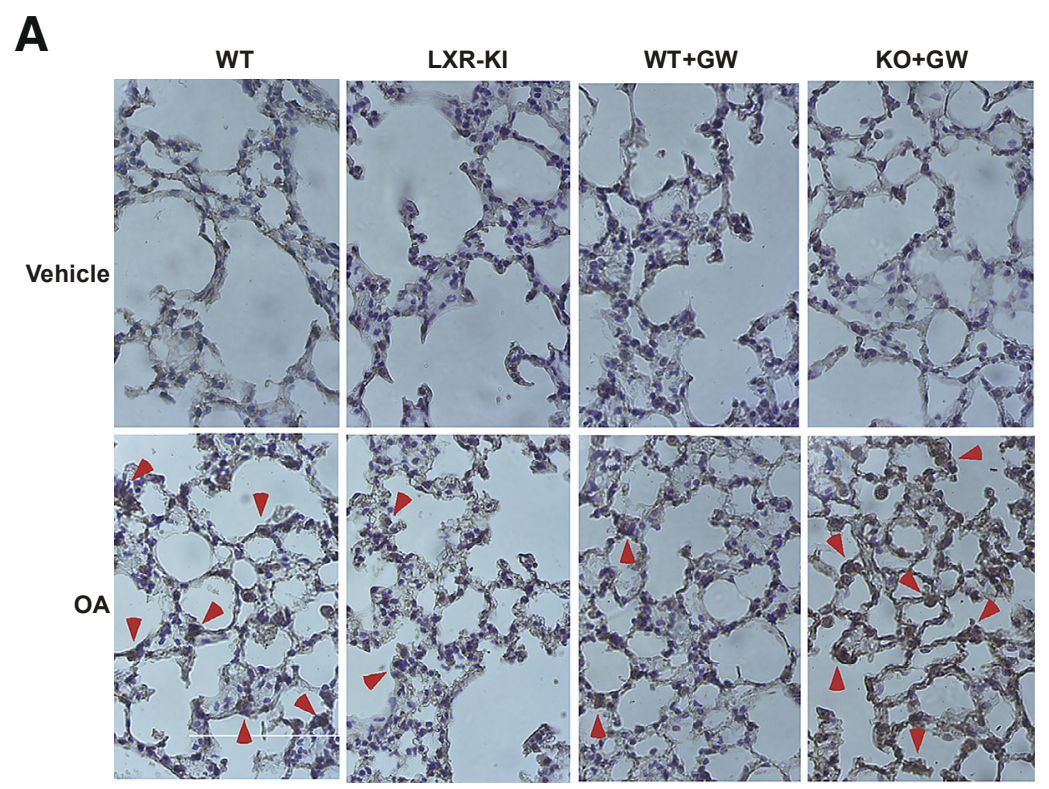

B

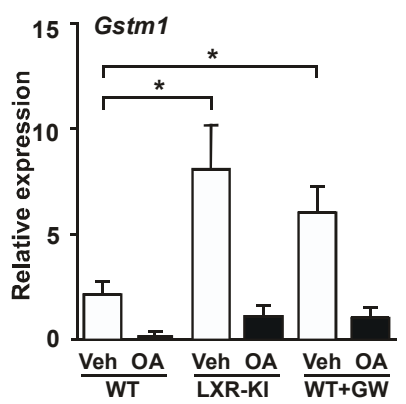

D

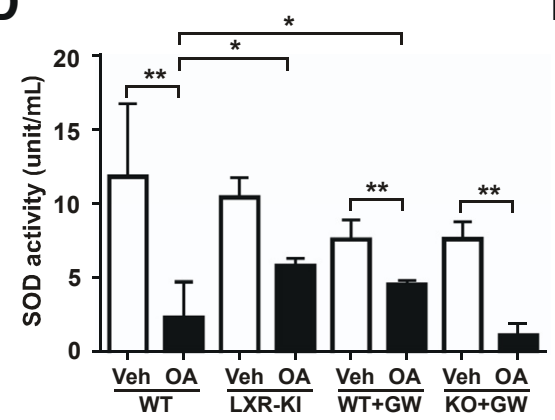

C

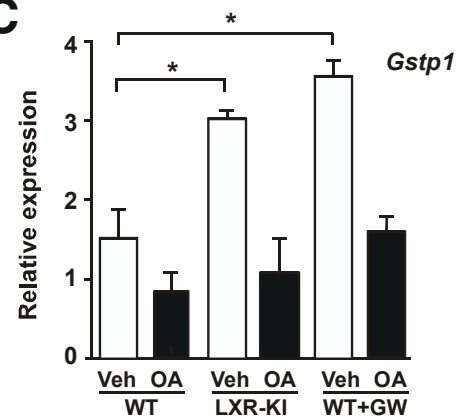

E

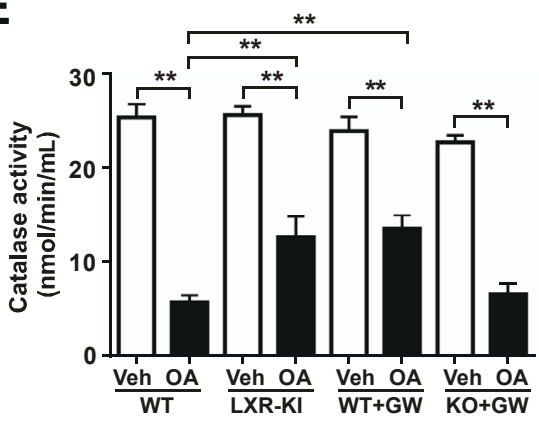

Figure 5 The protective effect of liver $X$ receptor (LXR) is associated with attenuation of oleic acid $(O A)$-induced oxidative stress and induction of antioxidant genes. A: Immunohistochemical staining of the lung paraffin sections using the anti-8-hydroxyguanosine antibody. Wild-type (WT), LXR knock-in (LXR-KI), and LXRDKO [knockout (KO)] mice received a single tail vein injection of vehicle (Veh) or OA. When necessary, WT and LXR-DKO mice were treated with GW3965 (GW; daily i.p. injections at $20 \mathrm{mg} / \mathrm{kg}$ ) for 7 days before being treated with Veh or OA. Mice were sacrificed 2 hours after the $0 \mathrm{~A}$ treatment and analyzed. Arrowheads indicate the positive staining. The lung expression of antioxidant genes Gstm1 (B) and Gstp1 (C) in WT and LXR-KI mice and GW3965-treated WT mice, in the absence or presence of $\mathrm{OA}$ treatment, was measured by realtime PCR. The activities of superoxide dismutase $(\mathrm{SOD} ; \mathbf{D})$ and catalase $(\mathrm{E})$ were measured in the lung homogenates. $n=5$ (each group, $\mathbf{B}$ and $\mathbf{C}$ ); $n=8$ (WT Veh group, $\mathbf{D}$ and $\mathbf{E}) ; n=11(\mathrm{WT}+0 \mathrm{~A}$ group, $\mathbf{D}$ and $\mathbf{E}) ; n=4$ (other groups, $\mathbf{D}$ and $\mathbf{E}$ ). ${ }^{*} P<0.05,{ }^{* *} P<0.01$. Original magnification, $\times 400(A)$. infiltration of neutrophils, production of proinflammatory cytokines, and oxidative stress.

We chose the OA model of ARDS, because the pulmonary injury responses induced by the i.v. administration of OA resemble the clinical ARDS. The OA model also mimics the critical pathogenesis of ALI, especially the marked accumulation of polymorphonuclear neutrophils and the release of inflammatory mediators. ${ }^{13}$ Moreover, the OA model provokes symptoms similar to those observed in human patients. Indeed, all of the hallmarks of ARDS, including inflammation, neutrophil activation, capillary leakage, tissue edema, and oxidative stress, were presented in the OA-treated WT mice. These pathological features were all significantly attenuated in the LXR-KI mice in which $\operatorname{LXR} \alpha$ was genetically activated, or in WT mice whose LXRs were pharmacologically activated by GW3965. The pulmonoprotective effect of GW3965 was abolished in the LXR-null mice, pinpointing that the GW3965 effect was mediated by LXRs. Sex-specific differences in pulmonary morbidity in humans are well documented. The mortality of ARDS was higher in male patients compared to female patients. ${ }^{28}$ In our model, both the male and female LXR-KI mice were protected from the OA-induced ARDS.

The anti-ARDS effect of LXR may have been contributed to by its positive regulation of antioxidant genes and the consequent relief of oxidative stress. The lung is susceptible to oxidative stresses that are derived from oxygen or inflammatory responses. ${ }^{9}$ The imbalance of oxidants and antioxidants 
plays an important role in the initiation and progression of ARDS. Oxidative stress also affects inflammatory responses by altering the activities of SOD, c-Jun N-terminal kinase, and mitogen-activated protein kinase and concentrations of cytokines. ${ }^{29}$ The expression of several antioxidant genes was induced in the LXR-KI mice and in GW3965-treated WT mice, consistent with our previous report that activation of LXR in mice induces antioxidant genes, leading to protection from lipopolysaccharide- ${ }^{16}$ and acetaminophen ${ }^{30}$ induced lung and liver tissue damages, respectively.

The large numbers of activated neutrophils in the lung in ARDS are generally considered as the major source of reactive oxygen species. ${ }^{13,31}$ Given that activation of LXR attenuated the OA-induced infiltration of neutrophils (Figure 3), it is possible that reducing the recruitment of neutrophils to lung by LXR activation may represent another potential mechanism by which LXR relieves ARDS. The inhibition of inflammation and infiltration of neutrophils by LXR has also been reported in experimental intracerebral hemorrhage and spinal cord trauma. ${ }^{32,33}$ Future studies are necessary to determine whether LXR activation in neutrophils affects their cytokine production, phagocytic function, and reactive oxygen species generation in OA-induced ARDS model.

In conclusion, we have uncovered a novel function of LXR in preventing ARDS. LXRs have been reported to have multiple potential therapeutic benefits, ranging from anti-atherosclerosis to anti-inflammation, ${ }^{34,35}$ macrophage survival and the innate immune response, ${ }^{36,37}$ and antiAlzheimer disease. ${ }^{38}$ Although avoiding the adverse effect of LXR activation, such as hepatic steatosis, ${ }^{39}$ remains a challenge, the anti-ARDS activity of LXR further warrants continued exploration of LXRs as therapeutic targets.

\section{Acknowledgments}

We thank Dr. David Mangelsdorf (University of Texas Southwestern Medical Center, Dallas, TX) for the liver X receptor DKO mice. We also thank Dr. Haibiao Gong (Fluidigm, San Francisco, CA) for his original creation of the VP-LXR knock-in mice and Dr. Gregg E. Homanics (University of Pittsburgh, Pittsburgh, PA) for his assistance in the creation of the VP-LXR knock-in mice.

Z.Z., D.X., and W.X. are the guarantors of this work and, as such, had full access to all of the data in the study and take responsibility for the integrity of the data and the accuracy of the data analysis.

\section{Supplemental Data}

Supplemental material for this article can be found at http://dx.doi.org/10.1016/j.ajpath.2016.06.018.

\section{References}

1. Dagvadorj J, Shimada K, Chen S, Jones HD, Tumurkhuu G, Zhang W, Wawrowsky KA, Crother TR, Arditi M: Lipopolysaccharide induces alveolar macrophage necrosis via CD14 and the P2X7 receptor leading to interleukin- $1 \alpha$ release. Immunity 2015, 42:640-653

2. Matthay MA, Ware LB, Zimmerman GA: The acute respiratory distress syndrome. J Clin Invest 2012, 122:2731-2740

3. Ashbaugh DG, Bigelow DB, Petty TL, Levine BE: Acute respiratory distress in adults. Lancet 1967, 2:319-323

4. Gordon D, Rubenfeld GD, Caldwell E, Peabody E, Weaver J, Martin DP, Neff M, Stern EJ, Hudson LD: Incidence and outcomes of acute lung injury. N Engl J Med 2005, 353:1685-1693

5. Han S, Mallampalli RK: The acute respiratory distress syndrome: from mechanism to translation. J Immunol 2015, 194:855-860

6. Matthay MA, Calfee CS: Therapeutic value of a lung protective ventilation strategy in acute lung injury. Chest 2005, 128:3089-3091

7. Ware LB, Matthay MA: Clinical practice: acute pulmonary edema. N Engl J Med 2005, 353:2788-2796

8. Bhatia M, Moochhala S: Role of inflammatory mediators in the pathophysiology of acute respiratory distress syndrome. J Pathol 2004, 202:145-156

9. Ware LB, Matthay MA: The acute respiratory distress syndrome. N Engl J Med 2000, 342:1334-1349

10. Lang JD, McArdle PJ, O'Reilly PJ, Matalon S: Oxidant-antioxidant balance in acute lung injury. Chest 2002, 122:314S-320S

11. Hussain N, Wu F, Zhu L, Thrall RS, Kresch MJ: Neutrophil apoptosis during the development and resolution of oleic acid-induced acute lung injury in the rat. Am J Respir Cell Mol Biol 1998, 19:867-874

12. Motohiro A, Furukawa T, Yasumoto K, Inokuchi K: Mechanisms involved in acute lung edema induced in dogs by oleic acid. Eur Surg Res 1986, 18:50-57

13. Matute-Bello G, Frevert CW, Martin TR: Animal models of acute lung injury. Am J Physiol Lung Cell Mol Physiol 2008, 295:L379-L399

14. Repa JJ, Mangelsdorf DJ: The liver X receptor gene team: potential new players in atherosclerosis. Nat Med 2002, 8:1243-1248

15. Collins JL, Fivush AM, Watson MA, Galardi CM, Lewis MC, Moore LB, Parks DJ, Wilson JG, Tippin TK, Binz JG, Plunket KD, Morgan DG, Beaudet EJ, Whitney KD, Kliewer SA, Willson TM: Identification of a nonsteroidal liver $\mathrm{X}$ receptor agonist through parallel array synthesis of tertiary amines. J Med Chem 2002, 45: 1963-1966

16. Gong H, He J, Lee JH, Mallick E, Gao X, Li S, Homanics GE, Xie W: Activation of the liver $\mathrm{X}$ receptor prevents lipopolysaccharide-induced lung injury. J Biol Chem 2009, 284:30113-30121

17. Smoak K, Madenspacher J, Jeyaseelan S, Williams B, Dixon D, Poch KR, Nick JA, Worthen GS, Fessler MB: Effects of liver X receptor agonist treatment on pulmonary inflammation and host defense. J Immunol 2008, 180:3305-3312

18. Gonçalves-de-Albuquerque CF, Silva AR, Burth P, de Moraes IM, Oliveira FM, Younes-Ibrahim M, dos Santos Mda C, D’Ávila H, Bozza PT, Faria Neto HC, Faria MV: Oleic acid induces lung injury in mice through activation of the ERK pathway. Mediators Inflamm 2012, 2012:1-11

19. Lesur I, Textoris J, Loriod B, Courbon C, Garcia S, Leone M, Nguyen C: Gene expression profiles characterize inflammation stages in the acute lung injury in mice. PLoS One 2010, 5:e11485

20. Lai JP, Bao S, Davis IC, Knoell DL: Inhibition of the phosphatase PTEN protects mice against oleic acid-induced acute lung injury. Br J Pharmacol 2009, 156:189-200

21. Henderson RF, Muggenburg BA: Use of bronchoalveolar lavage to detect lung injury. Curr Protoc Toxicol 2004, Chapter 18:Unit18.4

22. Li JW, Wu X: Mesenchymal stem cells ameliorate LPS-induced acute lung injury through KGF promoting alveolar fluid clearance of alveolar type II cells. Eur Rev Med Pharmacol Sci 2015, 19:2368-2378

23. Bradley PP, Priebat DA, Christensen RD, Rothstein G: Measurement of cutaneous inflammation: estimation of neutrophil content with an enzyme marker. J Invest Dermatol 1982, 78:206-209

24. Chow CW, Herrera Abreu MT, Suzuki T, Downey GP: Oxidative stress and acute lung injury. Am J Respir Cell Mol Biol 2003, 29: 427-431 
25. Cadet J, Douki T, Ravanat JL: Oxidatively generated damage to the guanine moiety of DNA: mechanistic aspects and formation in cells. Acc Chem Res 2008, 41:1075-1083

26. Cho HY, Reddy SP, Kleeberger SR: Nrf2 defends the lung from oxidative stress. Antioxid Redox Signal 2006, 8:76-87

27. Ganguly K, Depner M, Fattman C, Bein K, Oury TD, Wesselkamper SC, Borchers MT, Schreiber M, Gao F, von Mutius E, Kabesch M, Leikauf GD, Schulz H: Superoxide dismutase 3, extracellular (SOD3) variants and lung function. Physiol Genomics 2009, 37:260-267

28. Moss M, Mannino DM: Race and gender differences in acute respiratory distress syndrome deaths in the United States: an analysis of multiple-cause mortality data (1979-1996). Crit Care Med 2002, 30: $1679-1685$

29. Nakamura $T$, Nakamura $H$, Hoshino $T$, Ueda S, Wada H, Yodoi J: Redox regulation of lung inflammation by thioredoxin. Antioxid Redox Signal 2005, 7:60-71

30. Saini SP, Zhang B, Niu Y, Jiang M, Gao J, Zhai Y, Lee JH, Uppal H, Tian H, Tortorici MA, Poloyac SM, Qin W, Venkataramanan R, Xie W: Activation of LXR increases acetaminophen clearance and prevents its toxicity in mice. Hepatology 2011, 54:2208-2217

31. Williams AE, Chambers RC: The mercurial nature of neutrophils: still an enigma in ARDS? Am J Physiol Lung Cell Mol Physiol 2014, 306: 217-230

32. Paterniti I, Genovese T, Mazzon E, Crisafulli C, Di Paola R, Galuppo M, Bramanti P, Cuzzocrea S: Liver X receptor agonist treatment regulates inflammatory response after spinal cord trauma. $\mathrm{J}$ Neurochem 2010, 112:611-624
33. Wu CH, Chen CC, Lai CY, Hung TH, Lin CC, Chao M, Chen SF: Treatment with TO901317, a synthetic liver $\mathrm{X}$ receptor agonist, reduces brain damage and attenuates neuroinflammation in experimental intracerebral hemorrhage. J Neuroinflammation 2016, 13:62

34. Castrillo A, Joseph SB, Marathe C, Mangelsdorf DJ, Tontonoz P: Liver X receptor-dependent repression of matrix metalloproteinase-9 expression in macrophages. J Biol Chem 2003, 278:10443-10449

35. Castrillo A, Joseph SB, Vaidya SA, Haberland M, Fogelman AM, Cheng G, Tontonoz P: Crosstalk between LXR and toll-like receptor signaling mediates bacterial and viral antagonism of cholesterol metabolism. Mol Cell 2003, 12:805-816

36. Joseph SB, Bradley MN, Castrillo A, Bruhn KW, Mak PA, Pei L, Hogenesch J, O'Connell RM, Cheng G, Saez E, Miller JF, Tontonoz P: LXR-dependent gene expression is important for macrophage survival and the innate immune response. Cell 2004, 119:299-309

37. Joseph SB, Castrillo A, Laffitte BA, Mangelsdorf DJ, Tontonoz P: Reciprocal regulation of inflammation and lipid metabolism by liver $\mathrm{X}$ receptors. Nat Med 2003, 9:213-219

38. Zelcer N, Khanlou N, Clare R, Jiang Q, Reed-Geaghan EG, Landreth GE, Vinters HV, Tontonoz P: Attenuation of neuroinflammation and Alzheimer's disease pathology by liver x receptors. Proc Natl Acad Sci U S A 2007, 104:10601-10606

39. Repa JJ, Liang G, Ou J, Bashmakov Y, Lobaccaro JM, Shimomura I, Shan B, Brown MS, Goldstein JL, Mangelsdorf DJ: Regulation of mouse sterol regulatory element-binding protein-1c gene (SREBP-1c) by oxysterol receptors, LXRalpha and LXRbeta. Genes Dev 2000, 14 : 2819-2830 